\title{
BENCHMARKING DEL MERCADO DE CAPITALES DE COLOMBIA Y CHILE PARA NO FINANCIEROS
}

\author{
Helver Ariel Anzola-Rodríguez \\ Escuela de Negocios, Gestión y Sostenibilidad \\ Programa de Especialización en Gestión Empresarial \\ Especialista en Gestión Empresarial \\ hanzola_15@hotmail.com
}

Mateo Pachón Rincón

Docente

Escuela de Ingeniería, Diseño e Innovación

mpachonr@poligran.edu.co

\section{Resumen}

El proceso de aproximación al mercado de capitales por parte de un no-experto se torna difícil por la gran cantidad de conceptos y la complejidad que involucra la interacción entre las diferentes partes interesadas. Esta es una de las razones del porqué se genera abandono en los emprendimientos, ya que uno de los factores más importantes para afrontar los retos del nacimiento de una iniciativa se ve representado en la dificultad para acceder a la información y poder alcanzar los beneficios que un mercado de capitales desarrollado puede brindar al sector real, donde además no se cuenta con políticas públicas que faciliten la integración al mismo. El presente artículo parte del análisis del mercado de capitales chileno y el colombiano para llevar la síntesis de su función a personas sin formación en finanzas.

Se decidió establecer un paralelo entre Colombia y Chile de uno de los medios más utilizados para la financiación de proyectos como es el mercado de capitales, donde se busca conocer bondades y características del mismo con la finalidad de comprenderlo y aceptarlo cuando sea conveniente. Se comienza con un análisis de datos, posteriormente se realiza un estudio comparativo y finalmente se dan resultados asociados a los hallazgos sobre las generosas características del mercado de capitales en Chile.

Dentro de las economías latinoamericanas se debe resaltar la manera como Chile viene construyendo un camino que lo ha llevado a ser el país de la región más atractivo para la inversión 
y donde mayor probabilidad de éxito pueden llegar a tener los nuevos negocios, tan solo al consultar la página del Ministerio de Hacienda se encuentra un vínculo en la pantalla principal donde se evidencia la importancia que le da el gobierno como "piedra angular del sistema económico", por otra parte al consultar la página del Ministerio de Hacienda en Colombia no se encuentra mayor información y la búsqueda arroja pocos resultados. El estudio comparativo permite vislumbrar oportunidades en el mercado de capitales que sirve como punto de encuentro entre quienes desean invertir y quienes necesitan financiamiento, este es el lugar donde los excedentes de liquidez desempeñan su mejor papel atrayendo un retorno importante y a su vez financiando iniciativas que pueden ser exitosas en el futuro.

El tema tratado en este artículo ha sido abordado en varias ocasiones por especialistas que cuentan con suficiente información y resultados comprobados de sus investigaciones, por tal razón el objetivo principal no es tratar de igualar las conclusiones de reconocidos personajes económicos de la nación, sino colocar parte de esas mismas conclusiones en un lenguaje accesible para cualquier persona, es decir en palabras un poco más comprensibles y que permitan desarrollar interés por el Mercado de Capitales como siento que me ocurrió.

Palabras Clave: abandono de emprendimientos, mercado de capitales, excedentes de liquidez, retorno importante, piedra angular 


\section{Introducción}

Hay múltiples evidencias de las bondades de un mercado de capitales estructurado y también de su alto desempeño como desarrollador del sector real mediante la transferencia de recursos para ampliación o mejora de proyectos nuevos o ya existentes. Por lo cual es necesario que las políticas del gobierno permitan clarificar las normas de participación y los interesados tengan pleno conocimiento de las implicaciones de estar involucrados (Córdoba Garcés \& Molina Ungar, 2017)

Es cierto que el mercado de capitales ha crecido y la primera misión trajo resultados contundentes con respecto al incremento de la capitalización bursátil, la emisión de bonos y democratización de la propiedad accionaria de las empresas estatales, sin embargo viene estancado y necesita un nuevo diagnóstico (Rodríguez, 2017). El desarrollo del mercado de capitales permite reducir el número de personas bajo la línea de pobreza y aumentar los ingresos de quienes menos perciben (Banca y Bolsa, 2010).

En Colombia se utiliza el mercado de capitales de manera limitada y la banca privada es el medio más frecuente, los inversionistas prefieren compañías con bajo riesgo y se quedan por fuera empresas de desarrollo medio a las cuales busca impulsar el mercado de capitales (Pontificia Universidad Javeriana, 2016). El desarrollo del mercado accionario tiene potencial para convertirse en generador de riqueza a largo plazo de las compañías colombianas por lo que se necesitan dinámicas favorables para su crecimiento (Cardona, Andrés Octavio;, 2017).

El mercado de capitales es esencial para asignar eficientemente los recursos de ahorro y para repartir riqueza entre la población, tomando un importante rol como columna vertebral del sistema económico (Arrau P, 2016), la economía y el mercado de capitales van de la mano y existe una relación directa en el desarrollo de ambos (Bertolino, 2017).

El Mercado de Capitales es piedra angular del sistema económico donde las personas y empresas acuden como medio de ahorro, protección y financiamiento. La existencia de un mercado sano y competitivo está directamente relacionada con el crecimiento económico del país (Ministerio de Hacienda, Gobierno de Chile).

Para una sociedad es indispensable contar con un mercado de capitales grande ya que este actúa como “corazón” de la economía (Berenstein, 20006).

La importancia de encontrar una contraparte entre los que quieren invertir y quienes necesitan financiación, resulta más difícil si no existe un punto de encuentro. De ahí, la necesidad de un mercado organizado que contribuya al traspaso de recursos (Comisión para el Mercado Financiero).

El buen desempeño de todo el conjunto es lo que conlleva crecimiento eficiente de la economía, reduciendo el riesgo y los costos, movilizando la producción de bienes y servicios para así poder crear nuevos empleos. (Solano Ramirez, Ernesto;, 2007). 
La oferta o emisión de títulos necesita un mayor impulso ya que la percepción de pequeños y medianos empresarios es de dificultad y alto costo al querer pertenecer a la bolsa de valores. (División Técnica de Corredores Asociados, 1996).

\section{Caso de estudio}

Cuando un colombiano llega a Chile, encuentra una sociedad muy particular donde el taco no es una tortilla sino el embotellamiento vehicular, donde la palta o aguacate se utiliza en la mayoría de comidas, donde puedes tomar agua de la llave pero el sabor es terrible, donde no hay novios y novias pero si hay pololos y pololas y se pololea, donde es mejor dormir con pijama por si en la noche hay un temblor y debes salir de prisa, donde el pisco es chileno y no peruano, donde los mejores vinos del planeta se producen en esos valles y no hay lugar a discusión.

Adicionalmente se encuentran varios términos propios que en ocasiones pueden hacer perder el hilo de la conversación; también hay una sociedad que a pesar de estar ubicada en el denominado cinturón de fuego del pacífico y tener una actividad sísmica sin comparación con otro lugar de este planeta, se arriesga con construcciones de vanguardia como las 50 torres de oficinas estándar premium denominadas Sanhattan que son el distrito financiero de la capital del país y se puede apreciar en su totalidad desde el Sky Costanera que es el mirador del piso 61 y 62 en Costanera Center, y que por cierto es la torre más alta de Latinoamérica con 300 metros de altura y ascensores de última generación que recorren 7 metros por segundo con capacidad máxima de 45 personas y que se puede apreciar en la siguiente imagen:

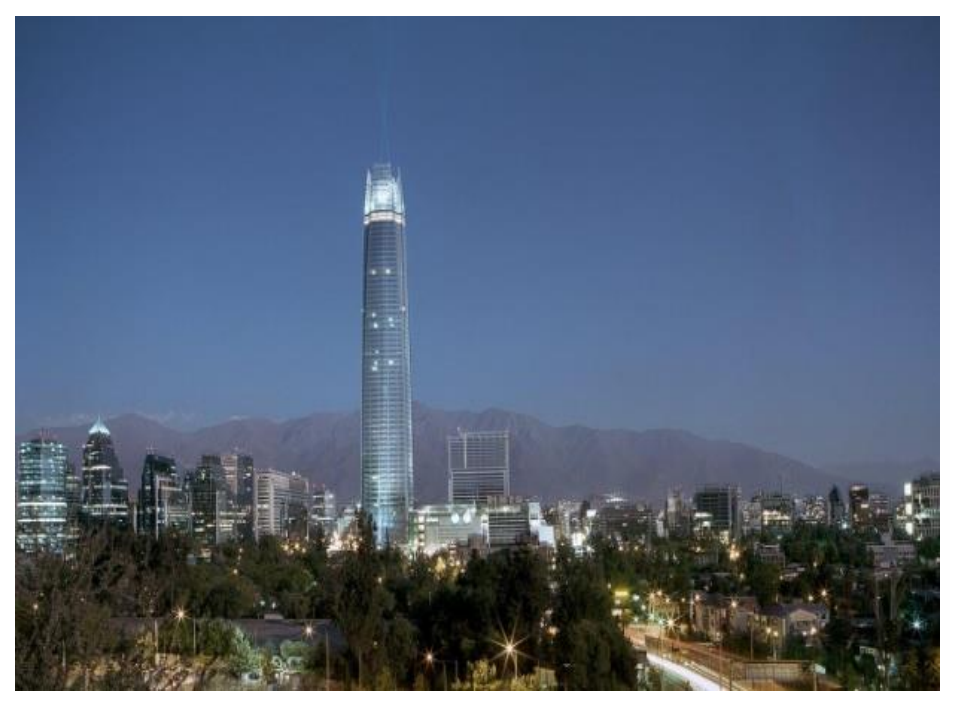

Imagen 1: Torre Costanera

Fuente: (Sky Costanera)

Adicionalmente como ellos dicen, se han vuelto expertos en construcciones subterráneas como el metro de Santiago y 19 túneles viales que ya están terminados más otros 9 que se 
están terminando de construir en todo el territorio con un costo aproximado de US\$15’000.000 por kilómetro.

\section{Se pueden estar preguntando: ¿Qué tiene que ver esto con el mercado de capitales?}

Al parecer tendrían que ser datos aislados y sin relación con el objeto de este artículo, pero tienen todo que ver porque el desarrollo vial y urbanístico de Chile es en parte resultado de un mercado de capitales bien estructurado, del cual hacen parte empresas como Besalco que tranza sus títulos en bolsa desde 1995, siendo representativa en Chile y los países donde opera, empresas como Parque Arauco con más de 12 centros comerciales y nuevos proyectos en marcha con presencia en Perú y Brasil, empresas como Paz Corp. con 150 proyectos y más de 25.000 viviendas para clase media, también hace parte activa el grupo SalfaCorp que se desempeña en sectores de la construcción, ingeniería e inmobiliaria, finalmente Socovesa que opera en bienes raíces y construcción, desarrollando obras de vivienda, unidades de vivienda, edificios corporativos, centros comerciales y oficinas, entre otros. (Bolsa Comercio Santiago)

Mientras tanto en Colombia, el documento "Misión del Mercado de Capitales" publicado en 1996 por Fedesarrollo, el Ministerio de Hacienda y el Banco Mundial fue la base para que se llevará a cabo la primer y hasta el momento única misión de este tipo dirigida en su momento por el hoy ministro Mauricio Cárdenas - con la finalidad de diagnosticar los aspectos relacionados con la hoja de ruta y fortalecimiento de los diferentes instrumentos que permitieran poner en marcha un mercado de capitales facilitador de inversión local y extranjera para el financiamiento de proyectos, emprendimientos o empresas ya existentes con planes de mejora o expansión.

La competencia por los recursos viene siendo global debido a la integración y educación financiera, al desarrollo de tecnología y al acceso a la información que permite conocer las oportunidades de inversión en el país donde se reside y también en las diferentes bolsas y mercados a nivel mundial.

Fortificar el mercado de capitales cobra tal importancia que en el año 2017 la Bolsa de Valores de Colombia (BVC) y el Depósito Centralizado de valores de Colombia (DECEVAL), acordaron fusionarse para hacer más eficiente la cadena de valor en la industria y fortalecer la competitividad del país en la región (en espera por aprobación de Superfinanciera).

Por otra parte el argumento de mejorar la competitividad del país, llevó en enero 2016 al presidente Juan Manuel Santos a anunciar como un "buen negocio" el hecho de haber vendido la más importante empresa generadora y comercializadora de energía del país, Isagen, con la finalidad de utilizar los 6.4 billones de pesos obtenidos por esa transacción para financiar las inversiones necesarias en infraestructura como las concesiones viales de 4G; pero en artículo del diario el colombiano (Correa, 2016) "De los 30 proyectos de vías $4 G$, solo tres tienen cierre financiero" se puede evidenciar que los ingresos por la venta de Isagen eran escasamente el $10 \%$ de lo necesario para financiar las etapas del ambicioso proyecto o definitivamente no iban a tener esa finalidad. 
Este mismo diario en artículo "El mercado de capitales es un ausente al financiar las vías 4G" (Arias Jimenez, 2015) nos plantea como Víctor Traverso Casagrande, ex director de la Corporación Andina de Fomento (CAF Colombia) insistía en que el mercado de capitales colombiano carecía de participantes y fue puntual en esta afirmación:

“Todo lo que busca el Gobierno, financiar 50 billones de pesos para ejecutar las obras, es algo que se puede hacer, pero ahora las reglas no son claras y hay impedimentos. Mientras en otros países una persona luego de 30 años de trabajo recoge su ahorro y compra una renta vitalicia, aquí eso no se puede hacer, es inexplicable" (Traverso, 2015).

En el mismo artículo Clemente del Valle, presidente de la Financiera de Desarrollo Nacional (FDN), consideró como válidas las inquietudes de expertos internacionales acerca de la falta de profundidad del mercado de capitales y hace la siguiente intervención:

"Necesitamos más actores y jugadores en ese mercado y algunas de esas cosas requieren regulación y otras aclaraciones" (del Valle, 2015).

En artículo del diario la república "En concesiones de cuarta generación, dos vías se han financiado con bonos" (Romero, 2016), nos muestran un buen ejemplo de cómo se pueden beneficiar emisores e inversores en el mercado de capitales bajo la emisión de bonos a largo plazo con atractivas tasas; la emisión producto de este artículo fue por 19 años con $8,25 \%$ de utilidad para financiar los proyectos Pacífico 3 que va desde La Virginia (Risaralda) hasta La Pintada (Antioquia) que contempla una inversión de $\$ 1,97$ billones y la troncal de Cartagena a Barranquilla que prevé una inversión de $\$ 1,80$ billones.

Este artículo menciona que las concesiones viales van a necesitar recursos por $\$ 62$ billones para vías, ferrocarriles, aeropuertos y puertos, distribuidos hasta el año 2022; también muestra la importancia que tiene la emisión de bonos para la financiación de proyectos viales en países como Chile con US\$4.000, Perú con US\$780 y los países de la Organización para la Cooperación y el Desarrollo Económico (OCDE) con US\$650.000 en emisión de bonos para el año 2016.

Al final del ejercicio con respecto al desarrollo de la estructura vial del país, se evidencia que las utilidades producto de estas inversiones son generosas, tienen una interesante extensión en el tiempo, hay respaldo nacional y confianza internacional pero no hay una presencia de actores locales diferentes a la banca tradicional. Entonces con un mercado de capitales desarrollado ¿hubiera sido posible que la nación conservara Isagen y por medio de la emisión de bonos se lograra la financiación de las vías de 4G?

Esta es una pregunta sin respuesta pero que se puede suponer.

Para generar un contexto de mayor comprensión se debe entender que el mercado de capitales es un punto de encuentro regulado y seguro entre quienes necesitan recursos de financiación y quienes tienen excesos de liquidez y están dispuestos a invertir a cambio de una mayor utilidad. Las empresas y entidades públicas que cotizan en bolsa encuentran disponibilidad de recursos para financiar sus proyectos donde el dinero de los inversores directos o que utilizan fondos de inversión como intermediarios, pasa a través del Mercado de Capitales hacia aquellos que lo necesitan para llevar a cabo sus objetivos, donde los inversionistas van a encontrar diferentes opciones a su disposición con condiciones de tiempo y riesgo que varían dependiendo de la utilidad que deseen obtener. 
Por lo tanto, se buscará a continuación dar un poco de claridad sobre los tipos de inversión más transadas, como son la renta fija, la renta variable y los derivados:

\subsection{Bonos de Inversión (Renta fija):}

Es un título de deuda que otorga derechos de cumplimiento de un emisor hacia un inversionista, son instrumentos emitidos por entidades particulares o entidades públicas como sociedades anónimas o el mismo estado; cuando un país necesita recursos puede promover diferentes acciones para el recaudo y puede ser a través de impuestos, a través de la emisión de efectivo que traería inflación o se puede financiar con deuda mediante títulos públicos o los denominados Bonos del estado.

Por ejemplo en la página del Ministerio de Hacienda de Colombia se encuentran dos noticias acerca de este tema y la primera ocurrió el 5 de junio de este año donde la nación colocó \$250 mil millones en Títulos de Deuda Pública (TES) a corto plazo con 4,700\% como tasa de interés y vencimiento el 12 de marzo de 2019 y recibió demanda por $\$ 316$ mil millones, es decir 1,26 veces el monto ofrecido, (MinHacienda, Gobierno de Colombia, 2018), la otra noticia ocurre el 6 de junio, un día después, donde la nación colocó $\$ 350$ mil millones en TES a 5 años con tasa 2,509\%, a 10 años con tasa $3.485 \%$ y 20 años con tasa $3,485 \%$ y recibió demanda por $\$ 967$ mil millones, es decir 2,76 veces el monto ofrecido (MinHacienda, Gobierno de Colombia, 2018).

Con respecto a la emisión de deuda pública como son los bonos de la nación, el mercado de capitales se encuentra bien desarrollado permitiendo al Estado endeudarse en moneda nacional que ayuda a reducir la vulnerabilidad por choques externos y donde las cifras son contundentes con respecto al crecimiento de este tipo de emisiones, por ejemplo en el año 2000 se transaban $\$ 14,6$ billones y en 2016 se alcanzó la cifra de $\$ 1.500$ billones; como se puede observar hay sobre demanda de los mismos que representa confianza en la economía del país. (Rodríguez, Segunda mano para el mercado de capitales, 2017)

\subsection{Emisión de Acciones (Renta variable):}

Son títulos emitidos por una empresa y que representan una fracción de la misma, donde el comprador adquiere derechos sobre el emisor teniendo en cuenta el porcentaje de participación de las acciones adquiridas dentro del total de acciones existentes, las cuales pueden moverse en valor hacia arriba o hacia abajo sin que haya un límite para dichas tendencias.

La emisión más grande e importante en la historia de la BVC fue hecha por Ecopetrol en el año 2.007 cuando se permitió la democratización accionaria de la compañía mediante la Ley 1118 del 27 de diciembre de 2006 por medio del mercado de capitales, dando acceso a las personas naturales que deseaban hacerse con una parte de la empresa que hasta el momento había sido propiedad exclusiva del Estado.

Fueron 32 departamentos y 1.104 municipios que lograron vincular alrededor de 350.000 accionistas que se hicieron con la colocación inicial abierta al público (IPO, por sus siglas en 
inglés) de $\$ 5,7$ billones equivalentes al 10,1\% de la propiedad de la empresa, los cuales han recibido en estos 10 años dividendos por $\$ 7,09$ billones.

Ecopetrol es el emisor con mayor capital bursátil con $\$ 68,4$ billones que representan el $20,5 \%$ del total de la BVC con corte a octubre 2017.

A pesar de los buenos dividendos por la característica de renta variable, esta acción tuvo una desvalorización importante en los años 2014 y 2017 por la sobreoferta de crudo que afectó directamente las acciones de compañías petroleras como Ecopetrol. (Acciones, 2017)

\subsection{Derivados (Contratos a futuro):}

Es un contrato entre dos partes donde se acuerda una transacción en el futuro quedando establecidas las cantidades, precio, calidad, referencias y todas aquellas características explícitas que clarifiquen el compromiso de quienes intervienen.

El objetivo es la protección y no la ganancia de un subyacente, donde este subyacente es el producto derivado del contrato; que pueden ser acciones, títulos de renta fija, divisas, materias primas, entre otros. (Bolsa de Valores de Colombia)

El objetivo es acabar con la incertidumbre y el devenir de los precios a lo largo de un periodo de tiempo; por ejemplo:

Un explorador de petróleo hace un hallazgo, pero tiene incertidumbre sobre los precios del barril en el futuro, por lo que decide negociar con una contraparte la producción que tendrá y el precio al que venderá, donde uno se compromete a entregar la cantidad contratada por el precio establecido con anterioridad, y a su vez la otra parte se compromete a recibir dicha cantidad y pagar el valor del acuerdo.

\section{Análisis Comparativo}

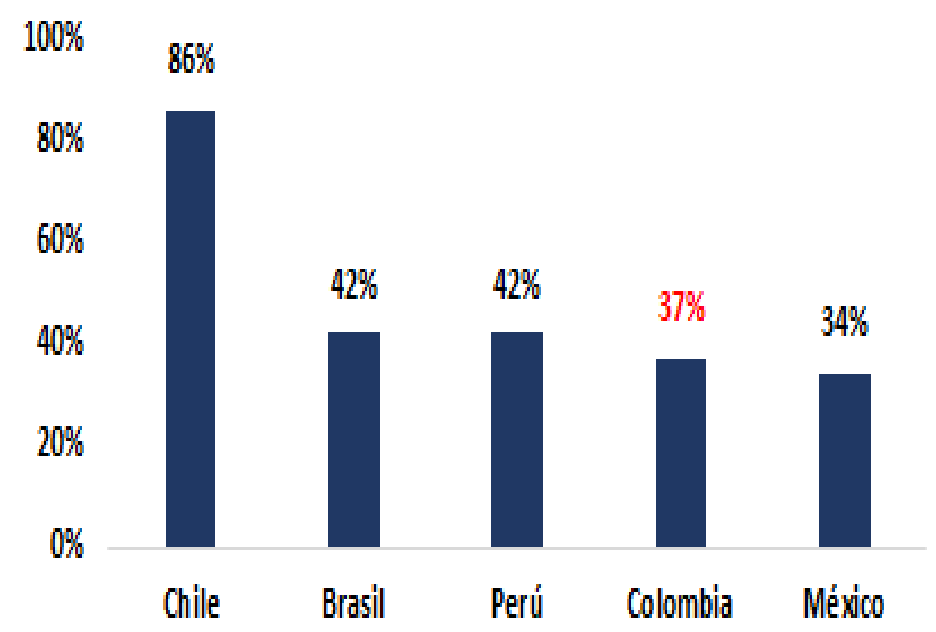

Figura 1: Capital bursátil como \% del PIB para 2016 (Córdoba Garcés \& Molina Ungar, 2017) 
Colombia no tiene ingreso de nuevas compañías a la bolsa de valores desde el año 2.013 y en total son menos de 80 las que tienen participación activa en la misma; esto puede tener varias interpretaciones como la falta de información acerca de los beneficios de pertenecer, el miedo a perder el control de la organización o la prevención por hacer pública la información correspondiente a los estados financieros.

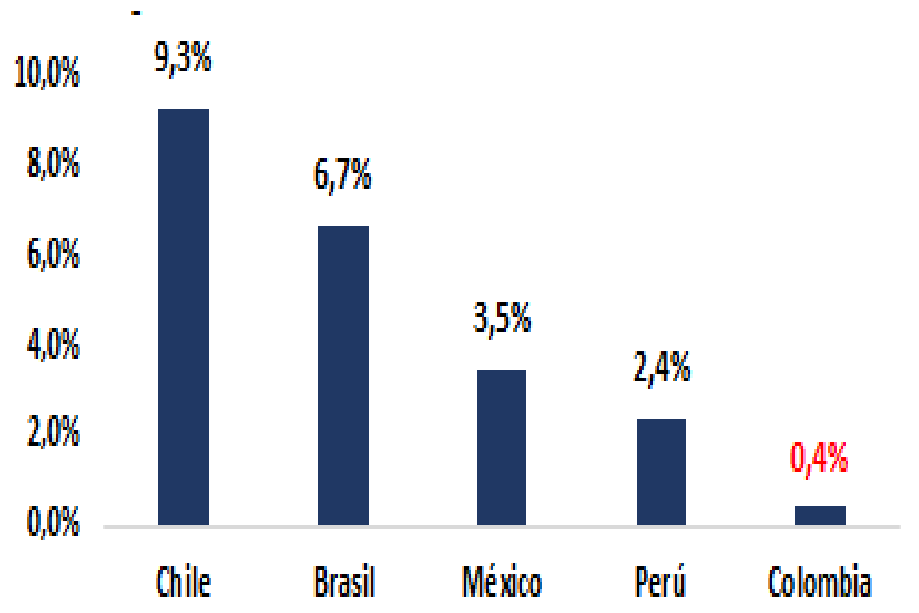

Figura 2: Valores de deuda en el mercado local del sector privado no financiero como \% del PIB para 2016

(Córdoba Garcés \& Molina Ungar, 2017)

La falta de regulación y estímulo para los empresarios hace que la financiación se haga principalmente con la banca tradicional mas no con la emisión de deuda, es cierto que al país lo mueven grandes capitales que no permiten la implementación de acciones que en determinado momento vayan a afectar sus intereses, pero este tipo de conducta genera estancamiento en el desarrollo económico por la influencia que tienen sobre el gobierno.

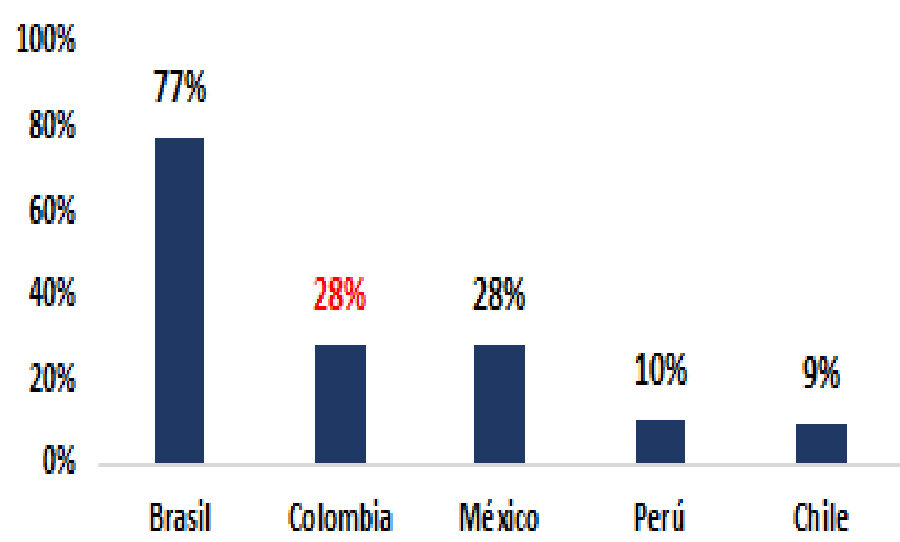

Figura 3: Valores de deuda en el mercado local del sector público como \% del PIB para 2016

(Córdoba Garcés \& Molina Ungar, 2017) 
El gobierno colombiano es activo en la emisión de deuda para financiar sus proyectos por medio del mercado de capitales, este segmento es uno de los más profundos y desarrollados de Latinoamérica donde los inversionistas nacionales y extranjeros financian con excedentes de liquidez las iniciativas del sector público, las cuales tienen sobre demanda al momento de estar disponibles; este resultado debería ser razón suficiente para que el gobierno implemente medidas que permitan al sector privado acceder a este tipo de financiamiento.

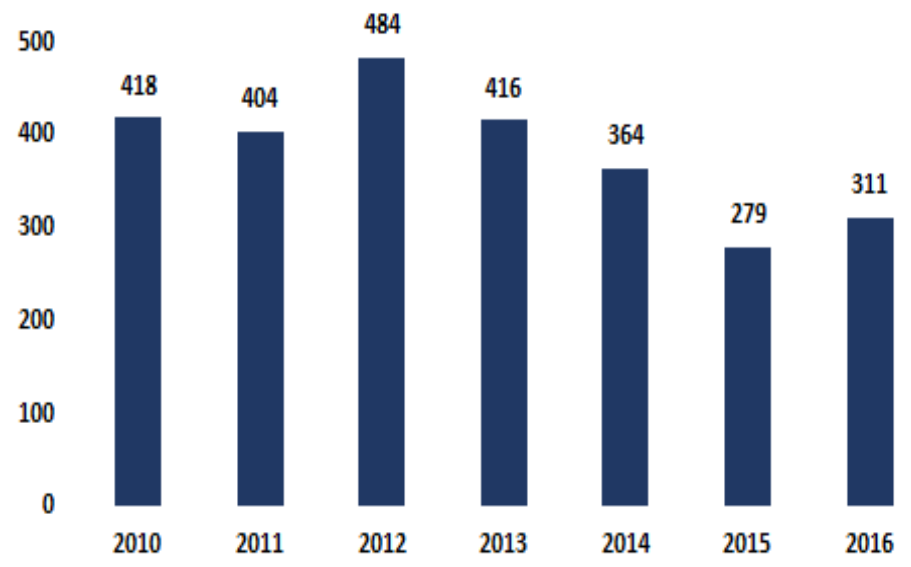

Figura 4: Capitalización bursátil para Colombia en trillones de pesos (Córdoba Garcés \& Molina Ungar, 2017)

Los descensos en la capitalización bursátil durante los últimos 5 años muestran que no hay suficientes estímulos para que las empresas se vinculen y hagan una primer emisión abierta al público de acciones, representando un retroceso significativo de la liquidez y en la cantidad de emisores de valores. Por ende, se limita el acceso al mercado de capitales.

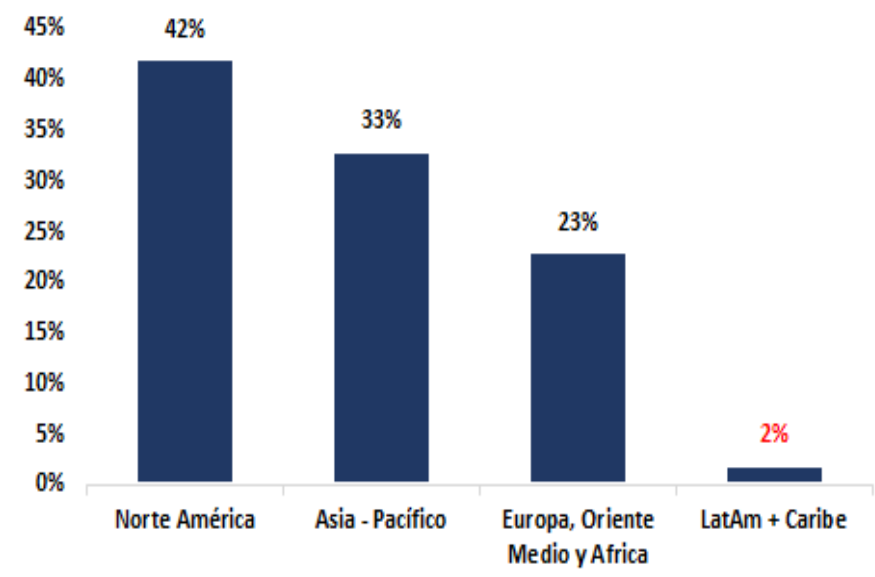

Figura 5: Participación en la capitalización bursátil de mercados de capitales mundiales para 2016

(Córdoba Garcés \& Molina Ungar, 2017) 
América Latina participa con el 9\% de la población mundial sin embargo el aporte al PIB está por debajo de ese porcentaje y el volumen transado en acciones solo alcanza el $0.8 \%$ del total, donde Brasil y México son las economías más representativas y dejan con participaciones mínimas a países como Colombia.

\section{Conclusiones}

Durante el mes de mayo del año en curso tuvo lugar uno de los debates por la presidencia de la república, donde el candidato y hoy electo presidente de la nación hizo la siguiente pregunta a uno de sus contendores:

"Doctor S, uno de los temas más importantes para el emprendimiento de un país es el desarrollo de los mercados de capital, Colombia tiene menos de 80 empresas listadas y son muy pocas las que transan más de 5 millones de dólares, ¿Qué piensa usted hacer para desarrollar los mercados de capital en Colombia?"

Para los conocedores del tema el candidato que debía responder no tuvo una acertada participación y esto generó varias reacciones en redes sociales e incluso en el diario La República mediante el titular "El erróneo desprecio al mercado de capitales" (Rodríguez, El erróneo desprecio al mercado de capitales, 2018).

Este se está realizando un empalme entre el equipo del presidente saliente y el equipo del presidente electo que a su vez está conformado por varios especialistas en el tema de este artículo y quienes lo abordan constantemente en sus columnas de opinión e intervenciones en conferencias.

Esta condición haría suponer que como mínimo la implementación de una segunda misión para el mercado de capitales estará dentro de la agenda del gobierno, ya que fue un tema puesto sobre la mesa por el electo presidente y donde su equipo de asesores tiene conocimientos suficientes para diagnosticar los resultados de la primera misión y a su vez identificar los retos de una futura misión.

El gobierno debe hacer planes de largo plazo y asimilar las buenas prácticas de países como Malasia que en 2001 lanzó su primer plan maestro para el mercado de capitales y le puso horizonte de 10 años para evaluarlo y robustecer en 2011; también Chile a realizado cuatro reformas estructurales desde el año 2001 para evaluar, adoptar las mejores prácticas internacionales, renovar políticas fiscales, tributarias y de competencia entre otras; para mantener el interés de los inversionistas actuales y atraer capital fresco con intención de permanencia.

Por lo tanto será necesario que el primer paso sea el de constituir un consejo consultivo o de asesoría para el Mercado de Capitales como el que viene funcionando en Chile desde el año 2.008 y que está conformado por reconocidos académicos, profesionales del sector bancario, del sector asegurador, de los fondos de pensiones, de los fondos de inversión y abogados especialistas en el ámbito corporativo y financiero; donde su principal objetivo sea el de promover activamente el desarrollo económico y el progreso social del país. 
Posteriormente recomendaría la implementación de cátedras relacionadas con fortalecer la educación financiera en los diferentes ambientes de formación, para que la inversión sea una cultura que permita establecer una participación activa en el desarrollo del país con portafolios diseñados para diferentes montos, dando acceso y confianza a las personas naturales y pequeños inversores.

Es importante fomentar el acceso y promoción de la información para que las pequeñas y medianas empresas puedan decidir cuál es la mejor opción de financiación con base en su estado actual.

Las pequeñas y medianas empresas no tienen excedentes importantes para la inversión, pero necesitan recursos y si las grandes empresas pueden financiar algunas de estas iniciativas mediante una figura relacionada al desarrollo de proveedores o por el simple hecho de obtener un incremento en sus utilidades complementado con un estímulo del gobierno, estaríamos dentro de una cadena de valor promoviendo beneficio compartido.

Es importante que el sector bancario tradicional comprenda que el mercado de capitales es complementario del mismo y no va reemplazarlo, aunque es natural que lo vean como competencia cuando los excedentes de capital o ahorros de pequeños inversores no sean depositados en la banca y se destinen para la financiación de proyectos que ellos mismos pudieron haber rechazado.

En ese momento el gobierno debe ser asertivo con los intereses de grupos económicos que pueden ver una disminución en sus utilidades con la implementación de medidas que motiven el desarrollo del mercado de capitales; llegando a sensibilizarlos para convencerlos de que ambos pueden y deben apoyar el sector real pero cada uno va desempeñar un papel protagónico en la vida de las empresas dependiendo del grado de madurez de la misma.

El gobierno necesita re evaluar la tasa impositiva que para 2017 fue del 40\% (34\% por impuesto a la renta y $6 \%$ por sobretasa sin contar parafiscales y demás gravámenes) que disminuye la competitividad del país al mantenernos por encima del promedio de los países miembros de la OCDE que para 2016 fue del 24,7\% y constituyen un alto impacto en las utilidades de nuestros empresarios.

Juan Pablo Córdoba menciona en el numeral 6 "Competitividad tributaria" (Córdoba Garcés \& Molina Ungar, 2017), que el país no es atractivo por el impacto de la tributación en la inversión y lo sustenta con el Reporte Global de Competitividad 2016-2017 generado por el Foro Económico Mundial que ubica a Colombia en el puesto 130, entre 138 países en la variable que evalúa los efectos de la tributación sobre la inversión; y en el puesto 133 en el indicador que mide el total de la tributación como porcentaje sobre las utilidades de las compañías.

Por último sería adecuado acatar las recomendaciones del Fondo Monetario Internacional (FMI, 2016) acerca del potencial de la integración regional como el Mercado Integrado Latinoamericano (MILA), que es una iniciativa gestada desde el año 2009 entre las economías de Chile, Colombia, Perú y México; con el objetivo de servir como plataforma para la negociación de acciones pero con el potencial de convertirse en columna vertebral de los países de la alianza del pacífico y posteriormente de los mercados financieros de la región con estrategias de desarrollo para enfrentar los retos que impone la coyuntura económica global. 


\section{Bibliografía}

Acciones. (2017). 10 años de la acción de Ecopetrol en la Bolsa de Valores de Colombia. Dinero, 1.

Arias Jimenez, F. (11 de Septiembre de 2015). El mercado de capitales es un ausente al financiar las vías 4G. El Colombiano, pág. 1.

Arrau P, P. (03 de Marzo de 2016). Obtenido de https://www.cepchile.cl/cep/site/artic/20160304/asocfile/20160304093235/10_arrau .pdf

Banca y Bolsa. (2010). ¿Por qué es importante el mercado de capitales? Dinero, 1.

Berenstein, M. (27 de Septiembre de 20006). e.news. Obtenido de 1

Bertolino, J. (04 de Septiembre de 2017). El Economista diario. Obtenido de https://www.eleconomista.com.ar/2017-09-rol-del-mercado-capitales/

Bolsa Comercio Santiago. (s.f.). Obtenido de http://www.bolsadesantiago.com/mercado/Paginas/detalleindicesbursatiles.aspx?ind ice $=$ CONST.$\% 26 I N M O B$.

Bolsa de Valores de Colombia. (s.f.). Bolsa de Valores de Colombia. Obtenido de https://www.bvc.com.co/pps/tibco/portalbvc/Home/Mercados/descripciongeneral/de rivados?action=dummy

Cardona, Andrés Octavio;. (23 de Noviembre de 2017). La importancia del mercado de capitales para las compañías. La República, pág. 1.

Comisión para el Mercado Financiero. (s.f.). Portal de Educación Financiera. Obtenido de http://www.svs.cl/educa/600/w3-article-511.html

Córdoba Garcés, J. P., \& Molina Ungar, E. (2017). Elementos para alcanzar el mercado de capitales que Colombia necesita: hoja de ruta y desafíos estructurales. Documentos CEDE No 70, 35 .

Correa, M. V. (04 de Julio de 2016). De los 30 proyectos de vías 4G, solo tres tienen cierre financiero. El Colombiano, pág. 1.

División Técnica de Corredores Asociados. (02 de Septiembre de 1996). Desarrollo del Mercado de Capitales en Colombia. El Tiempo, pág. 1.

MinHacienda, Gobierno de Colombia. (05 de Junio de 2018). Obtenido de www.minhacienda.gov.co/HomeMinhacienda/faces/SalaPrensa/Noticias/DetalleNot icia?documentId=P_MHCP_WCC-

116994\&_afrLoop=569416853290324\&_afrWindowMode=0\&_afrWindowId=8xjd 
eum5i_1\#!\%40\%40\%3F_afrWindowId\%3D8xjdeum5i_1\%26_afrLoop\%3D569416 $853290324 \% 26$ documentId\%

MinHacienda, Gobierno de Colombia. (06 de Junio de 2018). Obtenido de http://www.minhacienda.gov.co/HomeMinhacienda/faces/SalaPrensa/Noticias/Deta lleNoticia?documentId=P_MHCP_WCC-

117093\&_afrLoop=569359657290331\&_afrWindowMode=0\&_afrWindowId=8xjd eum5i_1\#!\%40\%40\%3F_afrWindowId\%3D8xjdeum5i_1\%26_afrLoop\%3D569359 $657290331 \% 26$ docu

Ministerio de Hacienda, Gobierno de Chile. (s.f.). Obtenido de

http://www.hacienda.cl/mercado-de-capitales/sobre-el-mercado-de-capitales.html

Pontificia Universidad Javeriana. (10 de Marzo de 2016). Obtenido de 1

Rodríguez, L. A. (24 de Mayo de 2017). Segunda mano para el mercado de capitales. La República.

Rodríguez, L. A. (17 de Mayo de 2018). El erróneo desprecio al mercado de capitales. La República, pág. 1 .

Romero, D. ". (15 de Febrero de 2016). En concesiones de cuarta generación, dos vías se han financiado con bonos. La República, pág. 1.

Sky Costanera. (s.f.). Obtenido de http://www.skycostanera.cl/es/fotos-y-videos/\#fotosvisitantes

Solano Ramirez, Ernesto;. (2007). Tu Guía Contable. Obtenido de http://www.tuguiacontable.org/app/article.aspx $? \mathrm{id}=465$ 